\title{
CLINICAL CHARACTERISTICS OF PMS CO-MORBID WITH MDD AND EFFECTIVENESS OF SSRIS IN ITS TREATMENT
}

\author{
R. Yakimova ${ }^{1,2}$, M. Stoimenova-Popova ${ }^{3,4}$, P. Chumpalova ${ }^{3,4}$, M. Pandova ${ }^{1}$, M. Stoyanova ${ }^{1,2}$ \\ ${ }^{1}$ University Multiprofile Hospital for Active Treatment in Neurology and Psychiatry "Sveti Naum" - Sofia, Bulgaria \\ 2Department of Psychiatry and Medical Psychology, Medical University - Sofia, Bulgaria \\ ${ }^{3}$ University Multiprofile Hospital for Active Treatment „Doctor Georgi Stranski“ - Pleven, Bulgaria \\ ${ }^{4}$ Department of Psychiatry and Medical Psychology, Medical University - Pleven, Bulgaria
}

\begin{abstract}
Background: Data on the clinical characteristics of premenstrual syndrome (PMS) with co-morbid major depressive disorder (MDD) are scarce. Although selective serotonin re-uptake inhibitors (SSRIs) are widely used to treat both PMS and MDD there is little information on their efficacy in PMS in patients with co-morbid MDD. Objective: To describe the clinical picture of PMS co-morbid with current depressive episode (DE) and evaluate its dynamics under a 6-month course of SSRIs treatment. Materials and methods: We present a longitudinal observational study, conducted in out- and inpatient conditions. Thirty-one women eligible to antidepressant treatment for a current $D E$ in the course of MDD were evaluated for PMS and those of them suffering from both conditions were re-evaluated twice during a 6-month medication intake period. Results: The pre-treatment clinical picture of PMS co-morbid with MDD was dominated by moderately to severely expressed mood swings, anxiety, fatigue, breast tenderness, palpitations, abdominal bloating, and headache. After six months of SSRIs intake the syndrome was characterised by nearly the same symptoms (with the addition of irritability and appetite changes) but mildly expressed. Conclusion: Untreated PMS co-morbid with MDD is characterised by mainly moderately severe psychological and around three times less commonly-somatic symptoms. SSRIs treatment alleviate both symptom types at month three and even further, although less pronouncedly at month six.
\end{abstract}

Key words: PMDD/PMS; major depressive disorder; co-morbidity; SSRI

Corresponding author: Milena Pandova, MD, University Multiprofile Hospital for Active Treatment in Neurology and Psychiatry "Sveti Naum" Ltd, Second Psychiatric Clinic, 1 "Dr. Lyuben Russev" Str., 1113 Sofia, Bulgaria, tel.: + 3598866830 53; e-mail milena.pandova@gmail.com

\section{INTRODUCTION}

ajor depressive disorder (MDD) is a common mental disorder with an unequal gender distribution [1]. In females the risk for MDD is approximately twice as high as in men $[2,3]$ but this gender difference encompasses only the reproduc- tive period of women [4] and especially the periods of substantial change in female hormonal levels [58]. This gives a reason to postulate the existence of relationship between premenstrual dysphoric disorder (PMDD) as a subtype of premenstrual syndrome (PMS) and affective disorders, especially puerperal depression and depressive episode (DE) in the 
course of MDD $[9,10]$. In fact, the close relationship between PMDD and depression is clearly reflected in its very categorisation in the fifth edition of the Diagnostic and Statistical Manual of Mental Disorders (DSM-5) along with the depressive disorders [11].

A high rate of co-morbidity between PMS/PMDD and DE in the course of MDD has already been documented on a number of occasions [12, 13]. PMDD is found much more frequently in women suffering from MDD [14] and between $30 \%$ and $70 \%$ of women with PMS or PMDD have suffered a previous DE in the course of MDD $[9,15,16]$. The incidence of depression is also higher among patients with PMS/PMDD than among healthy women $[17,18]$. Moreover, the frequency of depression in that population correlates positively with PMS severity [19].

Having in mind this close co-morbidity but also the similarity in the presentations of PMS and MDD we decided to examine the clinical picture of PMS when simultaneously present with MDD. We further evaluated its dynamics under a course of SSRIs intake.

\section{MATERIALS AND METHODS}

\section{Design}

The study was designed as observational despite including treatment as usual for a current depressive episode in the course of MDD. We assessed the clinical features, symptoms severity, and the ratio of somatic to psychological symptoms of PMS in women suffering from DE, as well as the evolution of the syndrome under selective serotonin re-uptake inhibitors (SSRIs) treatment. All diagnostic, rating, and therapeutic procedures were executed by a trained psychiatrist (first author) on the basis of the severity of the DE.

\section{Study population}

Forty-five (45) women with depressive episode in the course of MDD and accompanying PMS were enrolled. Fourteen (14) of them dropped out because of inaccurate completion of the questionnaire, noncompliance or preterm termination of treatment, failure to appear at a visit. The final sample consisted of 31 women. The study sample was recruited in both outpatient and inpatient conditions. Inclusion criteria were fertile age (18-50), regular menstrual cycle of 21-35 days duration, and a current DE in the course of MDD and exclusion criteria were lactation at present or in the past three months, hyster- and/or ovariectomy, and gynaecological conditions, use of hormonal products with potential effects on the physiological cyclicality of female sex hormones. Diagnoses were made according to the criteria of DSM-5
[11] by means of Mini-International Neuropsychiatric Interview (M.I.N.I.6.0) [21]. The participants were first informed about the symptoms of PMS and then invited to fill in a DSM-IV based questionnaire for a retrospective evaluation of premenstrual symptoms in the previous twelve months (visit 1 ). Those of them regularly suffering premenstrual symptoms during that time frame formed the group of patients with MDD and co-morbid PMS. The patients were reassessed at 3rd (visit 2) and 6th month (visit 3) following initiation of SSRI intake by the same questionnaire but evaluating their symptoms during just the last two menstrual cycles.

\section{Diagnostic instruments}

M.I.N.I.6.0. is a brief structured diagnostic interview, developed for the purpose of diagnosing DSM-IV and ICD-10 disorders. It has been validated against the Structured Clinical Interview for DSM (SCID), the Composite International Diagnostic Interview for ICD10 (CIDI) and a professional expert opinion [21] and is often used in clinical as well as research studies.

For evaluation of the presence and severity of PMS we used a questionnaire that was developed on the basis of the Premenstrual Symptoms Screening Tool (PSST) [22]. It comprises of twenty questions tapping on different symptoms of PMS and the level of functioning. We tested the somatic symptoms separately. For the correct completion of the questionnaire all symptoms need to be present regularly during the two-week period before menses for the last year (last two months for the follow-up visits). All symptoms identified by the patients need to be described as mild, moderate or severe. This evaluation is the basis for defining the severity of PMS. In the present study the criteria shown in Table 1 were used. Severe PMS corresponds to PMDD.

Table 1. Criteria for evaluation of PMS severity

\begin{tabular}{|l|l|}
\hline mild/moderate PMS & severe PMS (PMDD) \\
\hline $\begin{array}{l}\text { At least one question from } \\
1 \text { to } 4 \text { evaluated as mild/ } \\
\text { moderate }\end{array}$ & $\begin{array}{l}\text { At least one question from } 1 \text { to } \\
4 \text { evaluated as severe }\end{array}$ \\
\hline $\begin{array}{l}\text { At least four questions from } \\
1 \text { to } 19 \text { evaluated as mild/ } \\
\text { moderate }\end{array}$ & $\begin{array}{l}\text { At least four questions from } 1 \\
\text { to } 19 \text { evaluated as severe }\end{array}$ \\
\hline $\begin{array}{l}\text { Question } 20 \text { evaluated as } \\
\text { mild/moderate }\end{array}$ & $\begin{array}{l}\text { Question } 20 \text { evaluated as } \\
\text { severe }\end{array}$ \\
\hline
\end{tabular}

Abbr.: PMS: premenstrual syndrome; PMDD: premenstrual dysphoric disorder

Ethics approval was granted by the Ethics Committee of Medical Centre "Sveti Naum", Sofia, Bulgaria. 


\section{Statistical analyses}

The Statistical Package for Social Sciences (SPSS), version 13.0. was used to perform the statistical analyses of the raw data. Along with descriptive and frequency analyses we run Kolmogorov-Smirnov and Shapiro-Wilk tests for testing for normality of the distribution. These were followed by the Wilcoxon Signed Ranks Test for comparison of two related groups with non-normal distribution and by the McNemar Test when testing the variables' change dynamically. P-value below 0.05 was considered statistically significant.

\section{RESULTS}

The studied sample consisted of 31 women aged 25 to 49 years, mean 39.32 (SD 6.91). All patients were treated with SSRIs: citalopram - 16 patients, 15 of them were receiving $20 \mathrm{mg}$ citalopram per day, 1 patient $-30 \mathrm{mg}$ per day; fluoxetine -3 patients were treated with $20 \mathrm{mg}$ per day; paroxetine -5 patients $-20 \mathrm{mg}$ per day; escitalopram -7 patients, 1 of them was treated with $20 \mathrm{mg}$ per day, 6 were receiving 10 mg per day.

At visit 1 all psychological symptoms were broadly distributed some of them affecting all participants (Figure 1).

The same applies for the somatic symptoms with the exception of weight gain and joint pain (Figure 2).

Most of the symptoms were also most commonly moderately severe. Only anxiety and breast tension were experienced mostly as severe, and apathy, depressed mood, weight gain and muscle pain as mild. Most of the subjects suffered from moderately severe PMS (Figure 3).

Generally, the clinical picture of the untreated PMS co-morbid with MDD was dominated by psychological symptoms which were nearly three times more common than somatic ones (2.88) (Figure 4).

घisit 1 Visit 2 Visit 3

40

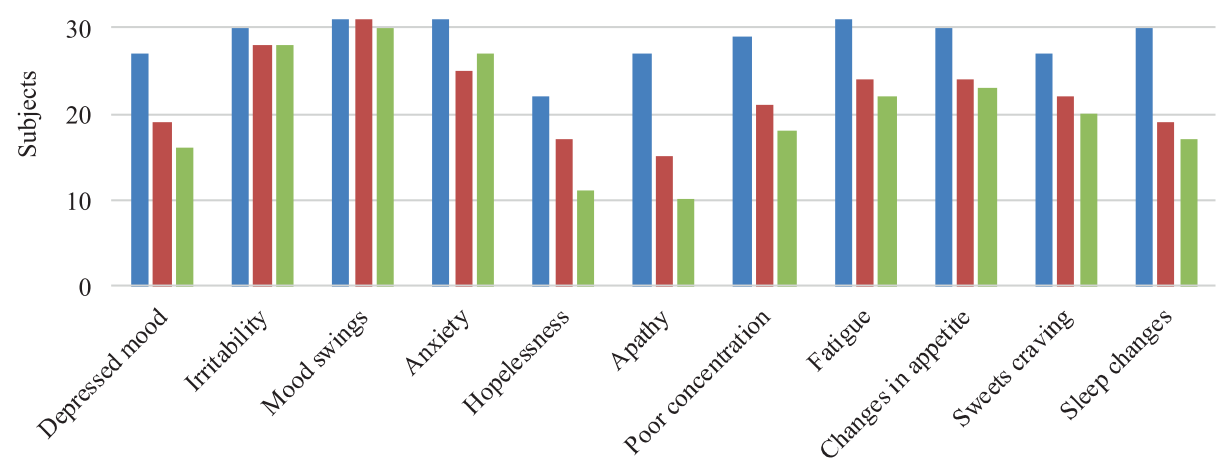

Fig. 1. Frequency of psychological symptoms of PMS $(n=31)$

- Visit 1 Visit $2 \square$ Visit 3

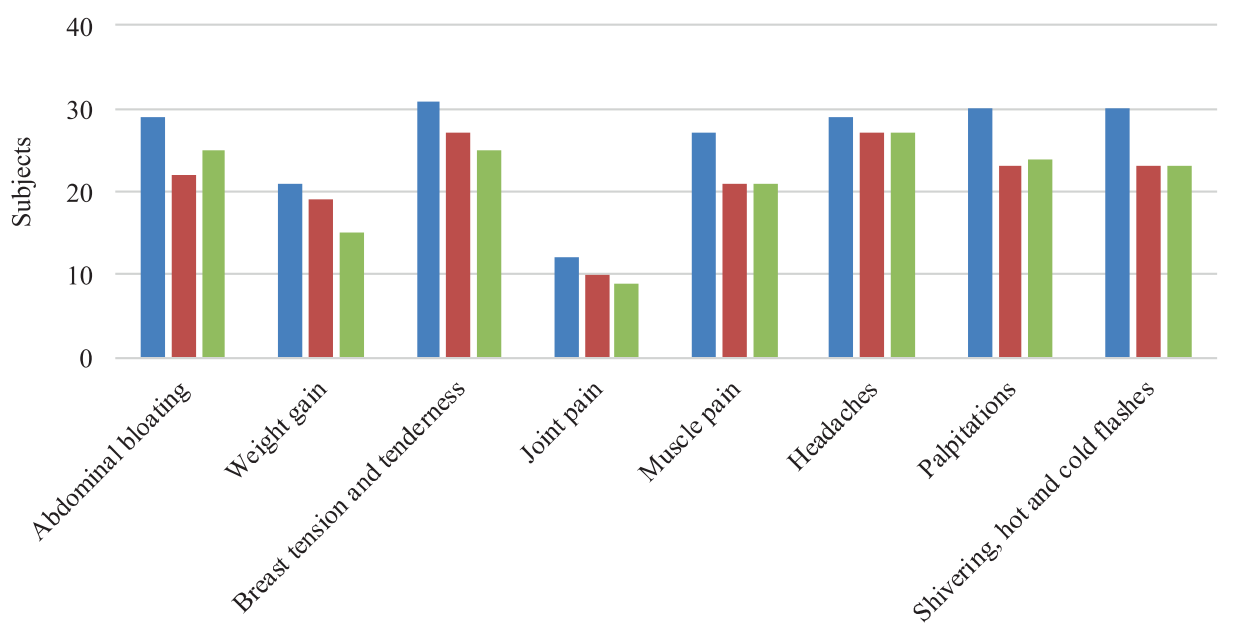

Fig. 2. Frequency of somatic symptoms of PMS $(n=31)$ 


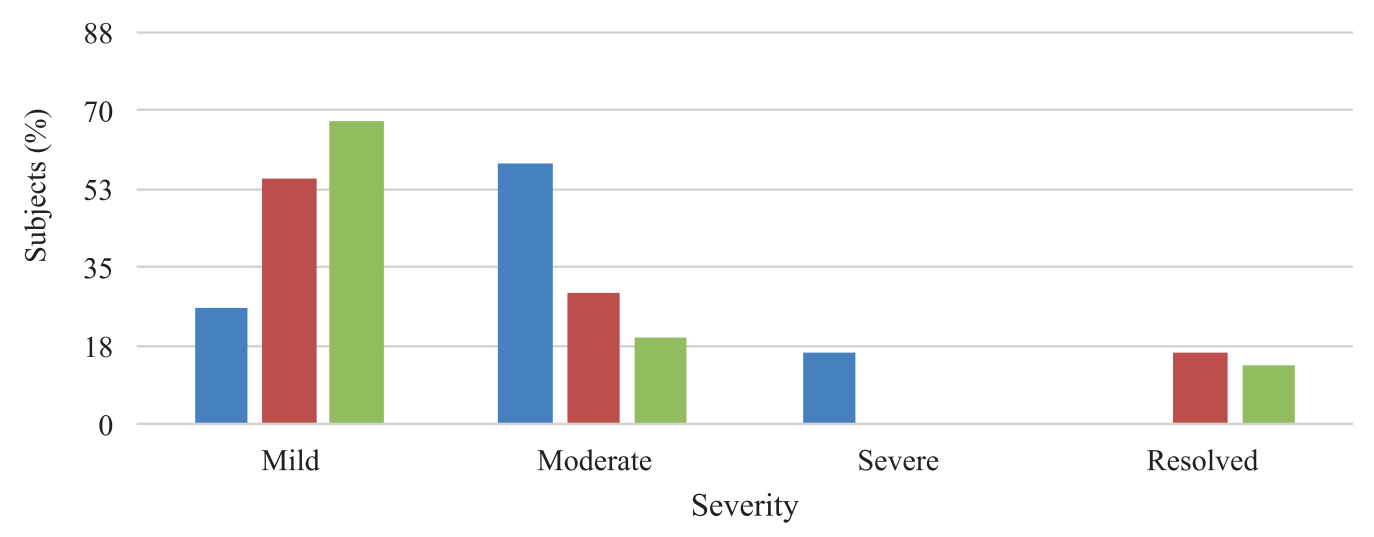

Fig. 3. Severity of PMS

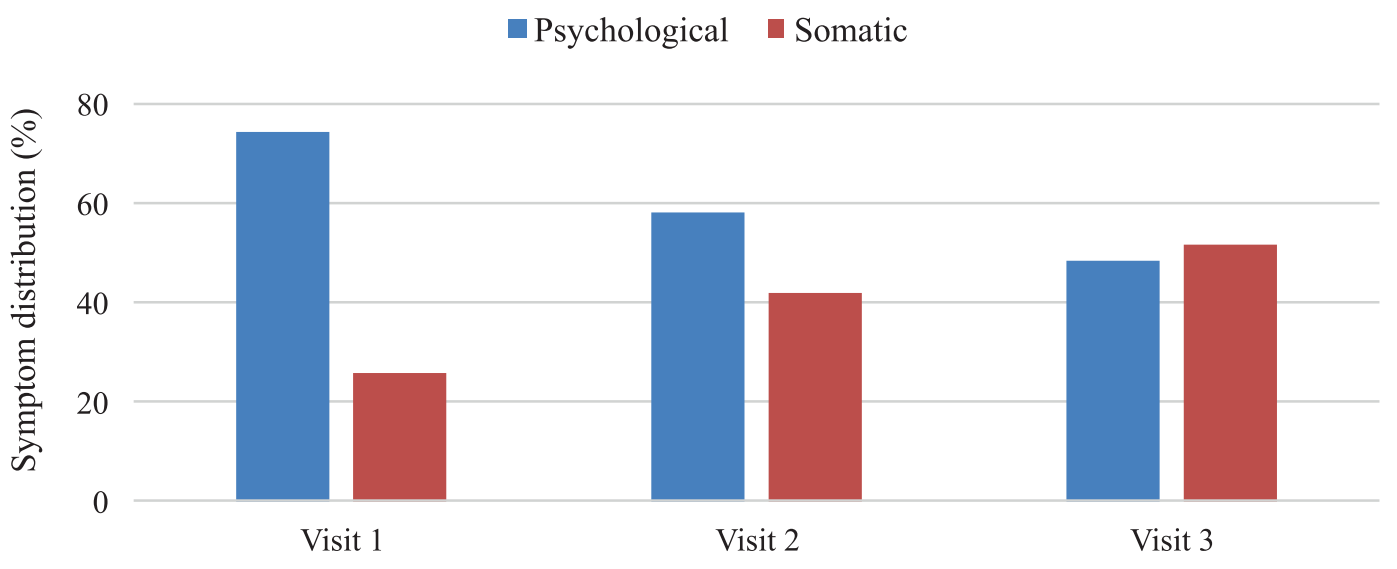

Fig. 4. Psychological vs somatic symptoms at each visit (\%)

The most common psychological symptoms remained unchanged during visits 2 and 3 , but their severity decreased to predominantly mild. Poor concentration and sleep changes were no longer that frequent as early as visit 2 . Similar dynamics was observed in the expression of the rest of the psychological symptoms (Figure 1). The change in the prevalence of all psychological symptoms at visits 3 compared to visit 1 was statistically significant (Table 2).

At visit 2 and visit 3 the most common somatic symptoms also remained the same (Figure 2). At visit 2 there was a statistically significant reduction in the frequency of all somatic symptoms with the single exception of weight gain. At visit 2 weight gain was also reduced in prevalence and severity but not to a statistically significant degree $(p=0.129)$. At visit 3 this was the only symptom which had changed significantly compared to the previous visit $(p=0.046)$ (Table 2). The prevalence of somatic symptoms at visit 3 compared to visit 1 was statistically significantly decreased, similarly to psychological symptoms (Table 2). At visit 2 psychological symptoms were still dominating the clinical picture of PMS with a ratio to somatic symptoms of 1.39 , while at visit 3 the prevalence of both types of symptoms almost equalised but with a slight predominance of somatic ones -0.94 .

At visit 2 there was statistically significant reduction in severity of the syndrome $(p<0.001)$ (Table 2). At visits 2 and 3 severe cases of PMS were lacking and there was a progressive rise in mild and decline in moderate cases. In certain proportion of the group the syndrome was fully resolved. There was a statistically significant difference in the syndrome severity between visit 1 and visit $3(p<0.001)$, but not between visits 2 and $3(p=0.660)$ (Table 2$)$.

\section{DISCUSSION}

The aim of the present study was to assess the clinical picture of untreated PMS co-morbid with MDD and describe the course of its response to SSRIs intake. Our results point that when a current $D E$ is present, psychological symptoms dominate the clinical picture of PMS. In our sample they were almost three times as common as somatic ones at the initial assessment (visit 1). Many of the psychological symptoms were in fact affecting all 
Table 2. Comparisons of symptoms' prevalence, severity of PMS, and psychological to somatic symptoms ratio between visits

\begin{tabular}{|c|c|c|c|c|c|c|}
\hline \multirow{2}{*}{ Psychological symptoms } & \multicolumn{2}{|c|}{ Visit $1 / 2^{*}$} & \multicolumn{2}{|c|}{ Visit $1 / 3^{*}$} & \multicolumn{2}{|c|}{ Visit $2 / 3^{*}$} \\
\hline & Z & $\mathrm{p}$ & z & $P$ & z & $\mathbf{P}$ \\
\hline Depressed mood & -3.418 & 0.001 & -3.779 & $<0.001$ & -2.999 & 0.003 \\
\hline Irritability & -3.314 & 0.001 & -3.448 & 0.001 & -1.000 & 0.317 \\
\hline Mood swings & -3.819 & $<0.001$ & -3.782 & $<0.001$ & -1.890 & 0.059 \\
\hline Anxiety & -4.039 & $<0.001$ & -3.908 & $<0.001$ & -0.060 & 0.952 \\
\hline Hopelessness & -2.546 & 0.011 & -3.520 & $<0.001$ & -3.000 & 0.003 \\
\hline Apathy & -3.380 & 0.001 & -4.208 & $<0.001$ & -2.271 & 0.023 \\
\hline Poor concentration & -4.564 & $<0.001$ & -4.562 & $<0.001$ & -1.633 & 0.102 \\
\hline Fatigue & -3.846 & $<0.001$ & -4.261 & $<0.001$ & -2.121 & 0.034 \\
\hline Changes in appetite & -3.412 & 0.001 & -3.960 & $<0.001$ & -2.530 & 0.011 \\
\hline Sweets craving & -2.696 & 0.007 & -3.345 & 0.001 & -2.333 & 0.020 \\
\hline Sleep changes & -4.225 & $<0.001$ & -4.289 & $<0.001$ & 0.000 & 1.000 \\
\hline \multirow{2}{*}{ Somatic symptoms } & \multicolumn{2}{|c|}{ Visit $1 / 2^{*}$} & \multicolumn{2}{|c|}{ Visit $1 / 3^{*}$} & \multicolumn{2}{|c|}{ Visit 2/3* } \\
\hline & Z & $p$ & Z & $\mathbf{P}$ & Z & $\mathbf{P}$ \\
\hline Abdominal bloating & -3.947 & $<0.001$ & -3.752 & $<0.001$ & -0.302 & 0.763 \\
\hline Weight gain & -1.518 & 0.129 & -2.251 & 0.024 & -2.000 & 0.046 \\
\hline Breast tension and tenderness & -3.134 & 0.002 & -3.779 & $<0.001$ & -0.913 & 0.361 \\
\hline Joint pain & -3.000 & 0.003 & -2.486 & 0.013 & -0.577 & 0.564 \\
\hline Muscle pain & -3.638 & $<0.001$ & -3.638 & $<0.001$ & 0.000 & 1.000 \\
\hline Headache & -4.117 & $<0.001$ & -4.345 & $<0.001$ & -1.000 & 0.317 \\
\hline Palpitations & -4.233 & $<0.001$ & -4.200 & $<0.001$ & -1.027 & 0.305 \\
\hline Shivering, hot and cold flashes & -4.179 & $<0.001$ & -4.284 & $<0.001$ & -0.302 & 0.763 \\
\hline PMS severity & -3.52 & $<0.001$ & -3.839 & $<0.001$ & -0.440 & 0.660 \\
\hline Psychological to somatic symptoms & \multicolumn{2}{|c|}{$0.063 \dagger$} & \multicolumn{2}{|c|}{$0.008 \dagger$} & \multicolumn{2}{|c|}{$0.250 \dagger$} \\
\hline
\end{tabular}

*Wilcoxon Signed Ranks Test; †Mc Nemar Test

or almost all participants. These results are in accordance with a previous report of greater prevalence of premenstrual psychological and behavioural than somatic symptoms among psychiatrically ill women [23].

Severely depressed women have also been shown to be mostly bothered by premenstrually occurring fatigue, food cravings, swelling, and weight gain and irritability, tension, mood swings, depression, lowered concentration and anxiety [24]. Premenstrual irritability of at least moderate severity was also a common symptom among perimenopausal depressed women [25]. This data largely overlaps with the most frequently reported symptoms in our sample of depressed women - mood swings, anxiety, and fatigue, irritability, changes in appetite and sleep, distractibility. Depressed mood and food cravings were also highly prevalent in our subjects, affecting nearly $90 \%$ of them. The only difference to our results concerns weight gain, which was the second rarest somatic symptom in the sample. But even though, it still affected far more than half of them $(67.7 \%)$. With regard to irritability, in our participants it was found to be not only highly prevalent but most commonly moderately and severely expressed as previously reported [25].

Our data further reveals an interesting tendency - for the gross majority of symptoms, both psychological and somatic, greater prevalence corresponded to greater level of severity. The only exceptions were depressed mood and apathy which despite being very common were predominantly mildly expressed.

So according to our results it seems that the clinical picture of PMS co-morbid with MDD is dominated by at best moderately severe anxiety, mood swings, fatigue, irritability, changes in appetite and sleep, distractibility, breast tenderness, palpitation, hot and cold flashes, abdominal bloating, and headache and mildly depressed mood, apathy, sweets craving, and muscle pain.

With regard to the change in symptoms under SSRIs treatment, our results showed an improvement in all symptoms following initiation of SSRIs intake. In the case of psychological symptoms there was a marked tendency towards reduction in severity to the point of full symptom resolution. For most of the symptoms this improvement was observable as early as visit 2 
(third month) of treatment initiation. Some of them (sadness, irritability, mood swings, anxiety, poor concentration, fatigue, carbohydrates craving, changes in sleep) reached their optimum improvement at that point and others (hopelessness, changes in appetite) continued to improve till visit 3 (sixth month). Irritability, anxiety and mood swings stood out as the most treatment resistant symptoms with very few cases of symptom resolution and prevalence of moderately severe forms till the sixth month.

In the case of somatic symptoms there was the same general tendency towards reduction in severity. A rise in the number of mildly expressed forms was observable but fewer cases of resolved symptoms were present compared to psychological ones. Furthermore, the change in prevalence and severity of a range of symptoms was optimally expressed as early as visit 2 (third month) with no or just minimal further gain till visit 3 (sixth month). The most difficult to treat symptom was apparently breast tension and tenderness which continued to prevail greatly at visit 2 as well as at visit 3 . Headache and abdominal bloating were also broadly distributed at visit 3 but with a clear drop in the number of severe cases to the extent that the proportion of mild ones became dominant.

This way while at the beginning of treatment psychological symptoms dominated over somatic ones, at visit 3 (end of the follow-up) this correlation was reversed.

A change in the severity of the syndrome was already marked at visit 2 but clearly expanded at visit 3 . During that time span could be outlined a tendency towards resolution of severe and drop in moderately severe cases and increase in mild and "remitted" ones.

Eventually, at visit 3 (sixth month) there was statistically significant reduction in frequency and severity of all symptoms. Psychological ones were ameliorated somewhat slower but more often completely and somatic ones, respectively faster but rarer fully. Hardest to improve were presumably mood swings, irritability, anxiety, headache, abdominal bloating, and breast tension and tenderness.

Based on these results it can be concluded that SSRIs are an effective treatment for PMS co-morbid with MDD with an observable response already at the visit 2 (third month).

A vast number of representative studies prove the efficacy of SSRIs for the treatment of PMS/PMDD. The results are consistent regardless of the dosages and treatment regimens utilised, although moderate dosages are generally related to greater effect sizes and higher response rates to treatment [26]. SSRIs are even considered an effective first line treatment for PMDD [27] and are the recommended therapy of choice by the latest consensus on the diagnosis and management of premenstrual disorder [28]. A reduction in symptoms of PMS/PMDD of at least $50 \%$ has been recorded in $51 \%$ to $63 \%$ of patients upon treatment with SSRIs [29], but the effect of treatment is observed no sooner than three cycles of administration with no further benefits of six cycles of treatment [30]. These findings overall corroborate our results especially with respect to somatic symptoms. We were able to demonstrate a pronounced and statistically significant improvement in the majority of psychological symptoms of PMS between visits 2 (third month) and 3 (sixth month). After month 3 (visit 2) there was a much more restricted effect in regard to somatic symptoms only one of which (weight gain) underwent an additional statistically significant improvement under the circumstances of longer medication intake.

But it seems that regardless of the duration of treatment the change in symptoms is reliable for the psychological ones like anger, mood swings, tension, depression, and irritability. The latter specifically is considered a prognostic marker of good reaction and swiftness of the response to SSRIs [29]. In our sample regardless of co-morbidity and greater severity of PMS all psychological symptoms were improved within the six-month treatment interval.

Nevertheless, with respect to the improvement of somatic symptoms data is a bit more contradictory [29]. SSRIs seem to be an effective treatment option for both somatic and behavioural symptoms but the results on the response of somatic ones is not definitive considering the lack of systematic evaluation of this type of symptoms in the design of many of the studies [27]. We tried to correct this flaw of previous research by examining somatic symptoms separately. This gave us the opportunity to definitely demonstrate an improvement in somatic along with psychological symptoms. Our data is consistent with the results of the major randomised controlled trials of SSRIs in the treatment of PMS, which clearly demonstrate an improvement not only in psychological (specifically irritability) but also in somatic symptoms (breast tension and abdominal bloating) [9, 13, 19, 22, 26]. It is yet possible that the improvement in certain group of symptoms is related to the regimen of medication administration - trials, comparing the effect of continuous versus intermittent medication intake associate the continuous one with a reduction in physical symptoms, and the intermittent one with improvement only in affective symptoms [29]. This question is a matter of further debate as both lack of considerable differences in the influence of either treatment regimen on symptoms [27], and an overall better effectiveness of the continuous treatment schedule have been reported [31]. In the presented study we used a continuous regimen and our results are a further confirmation of the effectiveness of SSRIs for the treatment of both somatic and psychological symptoms of PMS even when co-morbid with MDD. 


\section{CONCLUSIONS}

The typical clinical presentation of untreated PMS comorbid with MDD in Bulgarian women includes moderately severe symptoms, predominantly psychological ones. The syndrome itself is also most often moderately severe but still not rarely severe. It seems that a continuous regimen of administration of SSRIs can lead to a prominent improvement in psychological as well as somatic symptoms. It seems that somatic symptoms are improved somewhat faster but not fully, in contrast to psychological symptoms which continue to improve slower but more often to the point of full resolution.

Disclosure summary: The authors have nothing to disclose.

\section{REFERENCES}

1. Hsiao MC, Hsiao CC, Liu C. Premenstrual symptoms and premenstrual exacerbation in patients with psychiatric disorders. Psychiatry Clin Neurosci 2004; 58: 186-190.

2. Halbreich U. The etiology, biology, and evolving pathology of premenstrual syndromes. Psychoneuroendocrinology 2003; (Suppl 3): S55.

3. Vladimirova R. Specific mental health problems in women. Bulgarska nevrologichna i psikhiatrichna praktika 2004a; 3: 28-9.

4. Lasiuk G, Hegadoren K. The effects of estradiol on central serotonergic systems and its relationship to mood in women. Biological Research for Nursing 2007; 9(2): 147-160.

5. N-Wihlback A-C, Sundstrom-Poromaa I, Backstrom T. Action by and sensitivity to neuroactive steroids in menstrual cycle related CNS disorders. Psychopharmacology 2006; 186(6): 388-401.

6. Vladimirova R. Depression and pregnancy. Bulgarska nevrologichna i psikhiatrichna praktika 2004b; 1: 17.

7. Chaudron L, Klein M, Remington $P$ et al. Predictors, prodromes and incidence of postpartum depression. J Psychosom Obstet Gynecol 2001; 22(2): 103-112.

8. Freeman E, Sammel M, Rinaudo P, Sheng L. Premenstrual syndrome as a predictor of menopausal symptoms. Obstet Gynaecol 2004; 103: 960-966.

9. Critchlow D, Bond AJ, Wingrove J. Mood disorder history and personality assessment in premenstrual dysphoric disorder. J Clin Psychiatry 2001; 62(9): 688-693.

10. Wilson A, Henry DA. Meta-analysis Part 2 assessing the quality of published meta-analyses. Med J Aust 1992; 156(3): 173-187.

11. American Psychiatric Association. Diagnostic and statistical manual of mental disorders (5th ed.). Arlington, VA: Author. 2013.

12. Ito M, Matubara R. Premenstrual syndrome in women with mood and anxiety disorder. Clin Psychiatry 2006; 48: 750-763.

13. Kim D, Gyulai L, Freeman E et al. Premenstrual dysphoric disorder and psychiatric comorbidity. Archives of Women's Mental Health 2004; 7: 37-47.

14. Miyaoka $\mathrm{Y}$, Akimoto $\mathrm{Y}$, Ueda $\mathrm{K}$ et al. Fulfilment of the premenstrual dysphoric disorder criteria confirmed using self-rating questionnaire among Japanese women with depressive disorders. BioPsychoSocial Medicine 2011; 5: 5.

15. Cohen LS, Soares CN, Otto MW et al. Prevalence and predictors of premenstrual dysphoric disorder (PMDD) in older premenopausal women. The Harvard Study of Moods and Cycles. J Affect Disord 2002; 70: 125-132.
16. Soares C, Cohen L, Harlow MO. Characteristics of women with premenstrual dysphoric disorder (PMDD) who did or did not report history of depression: a preliminary report from the Harvard Study of Moods and Cycles. Journal of Women's Health and Gender Based Medicine 2001; 10(9): 873-878.

17. Adewuya A, Loto $O$, Adewumi T. Premenstrual dysphoric disorder amongst Nigerian university students: prevalence, comorbid conditions, and correlates. Archives of Women's Mental Health 2008; 11(1): 13-18.

18. Choudhari S, Rajshri, Inamdar S et al. A study of co-morbid depression and anxiety in pre-menstrual dysphoric disorder (PMDD) among undergraduate medical students: a descriptive study. Medica innovatica 2017; 6(1): 13-18.

19. Forrester-Knauss C, Stutz EZ, Weiss C, Tschudin S. The interrelation between premenstrual syndrome and major depression: Results from a population-based sample. BMC Public Health [Internet] 2011 Oct [cited 2018 Jun 28]; 11: 795. Available from: https://www.ncbi.nlm.nih.gov/pmc/articles/ PMC3209462/.

20. Treloar S, Heath A, Martin N. Genetic and environmental influences on premenstrual symptoms in an Australian twin sample. Psychol Med 2002; 32(1): 25-38.

21. Sheehan DV, Lecrubier Y, Sheehan KH et al. The Mini International Neuropsychiatric Interview (M.I.N.I.): The development and validation of structured diagnostic psychiatric interview for DSM-IV and ICD-10. J Clin Psychiatry 1998; Suppl 20: S22.

22. Steiner $M$, Macdougall $M$, Brown $E$. The premenstrual symptoms screening tool (PSST) for clinicians. Arch Womens Ment Health 2003; 6(3): 203-209.

23. Clare A. Psychiatric and social aspects of premenstrual complaint. Psychological Medicine. Monograph Supplement 1983; 4: 1-58.

24. Siegel JP, Meyers B, Dineen MK. Comparison of depressed and nondepressed women with severe Premenstrual Tension Syndrome. Psychother Psychosom 1986; 45: 113-117.

25. Richards M, Rubinow DR, Daly RC, Schmidt PJ. Premenstrual symptoms and perimenopausal depression. Am J Psychiatry 2006; 163: 133-137.

26. Marjoribanks J, Brown J, O'Brien PMS, Wyatt K. Selective serotonin reuptake inhibitors for premenstrual syndrome. Cochrane Database of Systematic Reviews [Internet] 2013 [cited 2018 Jul 10];7(6). Available from: http://onlinelibrary.wiley. com/doi/10.1002/14651858.CD001396.pub3/pdf .

27. Dimmock PW, Wyatt KM, Jones PW, O'Brien PM. Efficacy of selective serotonin-reuptake inhibitors in premenstrual syndrome: a systematic review. Lancet 2000; 356: 1131-1136.

28. Ismaili E, Walsh S, O'Brien PMS et al. Fourth consensus of the International Society for Premenstrual Disorders (ISPMD): auditable standards for diagnosis and management of premenstrual disorder. Arch Womens Ment Health 2016; 19: 953-958.

29. Halbreich U, O'Brien P, Eriksson E et al. Are there differential symptom profiles that improve in response to different pharmacological treatments of Premenstrual Syndrome/Premenstrual Dysphoric Disorder? CNS Drugs 2006; 20 (7): 523-547.

30. Brown J, O'Brien P, Marjoribanks J, Wyatt K. Selective serotonin re-uptake inhibitors for premenstrual syndrome. Cochrane Database of Systematic Reviews [Internet] 2009 Apr [cited 2018 Jul 08];2. Available from: doi: 10.1002/14651858. CD001396.pub2.

31. Shah NR, Jones JB, Aperi J et al. Selective serotonin reuptake inhibitors for premenstrual syndrome and premenstrual dysphoric disorder: a meta-analysis. Obstet Gynecol 2008; 111(5): 1175-1182.

Received: 26 March 2020, Accepted: 26 April 2020 